\title{
Oral Biofilms
}

\author{
Sonia B. Bhardwaj* \\ Dr Harvansh Singh Judge Institute of Dental Sciences and Hospital, Panjab University, \\ Chandigarh, India \\ *Corresponding author
}

\section{A B S T R A C T}

\begin{tabular}{|l|}
\hline Ke y w o r d s \\
Oral biofilms, Microbes, \\
Models of oral biofilms, \\
Oral diseases
\end{tabular}

\section{Introduction}

\section{Oral cavity}

The oral microbes comprise a complex community and that oral health or disease depends on the interface between the host and the microbial community. The oral ecosystem harbours more than 700 bacterial taxa. The bacteria colonize the hard surface of the teeth and the soft tissue of the oral mucosa. Different bacterial flora exists in the teeth, gingival sulcus, tongue, cheeks, hard and soft palate and tonsils (Dewhirst et al., 2010). The major genera found in the healthy oral cavity include Streptococcus, Veillonella, Granulicatella, Gemella, Actinomyces, Corynebacterium, Rothia, Fusobacterium, Porphyromonas, Prevotella, Capnocytophaga,
Biofilms are the form of life for great majority of oral bacteria and contribute to various oral diseases. To maintain oral health a balance between the human host and the microbes is essential. The excessive formation of oral biofilm results in intraoral diseases such as dental caries, gingivitis and periodontitis. The ability of pathogenic bacteria to form a biofilm increase their resistance to antibiotics. The most commonly pathogenic bacteria found in oral biofilms are methicillin resistant Staphylococcus aureus (MRSA), Staphylococcus epidermidis, Pseudomonas aeruginosa and Streptococcus mutans. The to emerge. The chapter focuses on oral pathobionts as commensals of oral biofilms and how they cause disease and various methods to study these oral biofilms.
Neisseria, Haemophilus, Treponema, Lactobacterium, Eikenella, Leptotrichia, Peptostreptococcus, Staphylococcus, Eubacterium and Propionibacterium (Wilson, 2005; Zaura et al., 2009; Em et al., 2010). Although the microbiome differs between the individuals, the microbiome per individual is constantly same in time (Badger et al., 2011).

\section{Development of oral biofilm}

Most of the oral bacteria are found in the oral biofilm which remains stable despite environmental changes (Marsh and Bradshaw, 1995). However, under certain conditions shifts in the composition of the dental plaque biofilm could lead to dental diseases such as dental caries and periodontal diseases. The bacterial composition in biofilm from a 
healthy peridontium is different from that of periodontitis (Lamont and Hajishengallis, 2014). The commensal bacteria in the mouth inhibit the colonization of pathogenic bacteria (Wade, 2013). However, the disruption in commensal biofilm results in the adherence and accumulation of pathogens in oral surfaces. The microbes adhere to the oral cavity and initiate the development of dental plaque biofilm. A highly organised sequence of events occurs. First, the adhesion of the microorganism on the surface of oral cavity takes place. The development of new plaque starts as soon as the tooth surface is covered with the protein film (pellicle). This process is very fast and takes place in minutes to form, after eruption of a new tooth or after removal of previous plaque (Hannig, 1999). The pellicle comprises of several molecules derived from the host and mainly contains mucins, proteins, agglutinins. Secondly, the multiplication of bacteria takes place.

The "early colonizers" bind to the pellicle covered tooth surface and mainly include streptococci and several gram-positive rods (Marloes et al., 2017). The early colonizers are then replaced by the" late colonizers". The biofilm is now dominated by Gram negative anaerobic bacteria. Fusobacterium nucleatum co-aggregates with other bacteria bridging organism by binding streptococci to pathogens such as Porphyromonas gingivalis, Treponema denticola, Prevotella intermedia and Tannerella forsythia (Kolenbrander et al., 2010).

Thirdly, the micro-colonies formed start to express a biofilm phenotype and excrete extracellular polymeric substance (EPS) leading to formation of three dimensionally spatially arranged mature biofilm community (Kolenbrander et al., 2002). The EPS present in the biofilm protects it from the mechanical flow of the saliva and other forces in the oral cavity (Stephens, 2002).

\section{Bacterial interaction in the oral biofilm}

The initial flora within few hours after birth is primarily Staphylococcus epidermidis and Streptococcus species mainly $S$. salivarius (Nelsen-Filho et al., 2013). The mature oral microbiome consists of hundreds of bacterial species. An ecologically balanced diverse microbiome which is in equilibrium with the host predominantly consists of Gram-positive facultative anaerobic bacteria, such as the Streptococcus and Actinomyces genera predominantly in healthy individuals (Jiao et al., 2014). Different studies have suggested a core oral microbiome of health (Fig. 1) which is different from the diseased one. A study done by Bik et al., (2010) on the largest set of near full length sequences in healthy individuals analysed 10 variables shared between 11 bacterial species. This supported the concept of both core and variable microbiome within the oral cavity.

The major genera in the healthy oral cavity includes Streptococcus, Veillonella, Granulicatella, Gemella, Actinomyces, Corynebacterium, Rothia, Fusobacterium, Porphyromonas, Prevotella, Capnocytophaga, Neisseria, Haemophilus, Treponema, Lactobacterium, Eikenella, Leptotrichia, Peptostreptococcus, Staphylococcus, Eubacterium and Propionibacterium (Dewhirst et al., 2010). Recently a study by (Mark Welch et al., 2016) utilizing combinational labelling and spectral imaging FISH has observed an oral microbiome termed as hedgehog structure consisting of nine taxa arranged in an organised spatial framework, which includes Corynebacterium, Streptococcus, Porphyromonas, Haemophilus / Aggregatibacter, Neisseriaceae, Fusobacterium, Leptotrichia, Capnocytophaga and Actinomyces. This hedgehog structure mainly consists of Corynebacterium as a multitaxon filament rich annulus and peripheral corn-cob structure. 
These filaments are comprised of Fusobacterium, Leptotrichia and Capnocytophaga whereas they are surrounded by Streptococcus, Porphyromonas and Aggregatibacter. Corynebacterium organisms are considered to be bridge species in regard to biofilm formation. Thus the oral biofilm is a network of multiple synergestic and antagonistic interactions generating microbial interdependencies and give biofilms a resistance from minor environmental disturbances. If these environmental disturbances exceed the threshold associated with health, a dysbiosis occurs between microbes causing the oral diseases (Marsh and Zaura, 2017).

\section{Distribution of genomes sequenced per phylum}

\section{Biofilm in dental caries}

Frequent intake of dietary sugars results in proliferation of bacteria such as Streptococcus mutans and Lactobacillus acidophilus creating an acidic environment causing the demineralisation of the enamel of the tooth (Marsh, 2010). The non-cariogenic plaque changes to cariogenic plaque causing the development of dental caries. Streptococcus sanguinis decreases after a micro-ecological imbalance being one of the causes resulting in dental caries. Other studies have shown that many other bacterial cariogenic species like Streptococcus mitis, Rothia, Actinomyces, Lactobacillus, Bifidobacterium and even fungal species like Candida albicans can account for a biofilm becoming cariogenic (Klinke et al., 2011). Recently a new cariogenic bacteria Scardovia wiggsiae has been found to be associated with dental caries (Tanner et al., 2011). The ecological changes which these organisms bring in the biofilm changing it to cariogenic by down regulating the main energy generating glycolytic pathway in order to be acid tolerant and also some bacteria undergo phenotypic changes in health and disease.

\section{Biofilm in periodontal disease}

Periodontal biofilms are formed by coaggregation of opportunistic microorganisms caused by diverse factors like poor oral hygiene. The periodontal tissue inflammation in the early stages is known as gingivitis and can be treated by removing the dental biofilm. The presence of dental plaques in gingival tissue and interactions of pathogens with host cells cause inflammation leading to periodontitis. Chronic periodontitis is now linked to systemic inflammatory diseases like artherosclerosis, infective endocarditis, diabetes, respiratory diseases and rheumatoid arthritis (RA) (Kim and Amar, 2006; Gaffen et al., 2014; Hajishengallis, 2015). In periodontitis there is proliferation of gram negative pathogenic bacteria like $P$.gingivalis, Spirochaetes like Treponema denticola in the dental plaque biofilm (Pihlshrom et al., 2005). The three bacterial species of the "red complex" Tanerella forsythia, $P$. gingivalis, $T$. denticola are the indicators of the periodontitis. A small rise in $\mathrm{pH}$ allows the periodontal pathogens to overgrow other microorganisms in the dental plaque (Marsh, 1994). Factors like smoking contribute to enhancement of colonisation of periodontal pathogens like Tanerella forsythia, P.gingivalis, T.denticola, P.intermedia, Parvimonas micra, Prevotella nigrescens and Campylobacter rectus (Shchipkova et al., 2010). Recent studies have shown that bacteria like Selenomonas have association with periodontal disease (Drescher et al., 2010) (Table 1).

\section{Diagnostic techniques to study oral biofilms}

The currently available models and techniques to study oral biofilms include: Saliva- Saliva only or its combination with selective media 
can be used. In multispecies biofilms the medium described by (Guggenheim et al., 2001) can be used. To this fluid universal medium (FUM) can be added like $\mathrm{N}$-acetyl Muramic acid for $T$. forsythia; $0.34 \mathrm{mM}$ hemin for $P$. gingivalis (Ammann et al., 2013).

\section{Support surfaces to study Oral Biofilm}

Inert surfaces like glass tube containing specific medium and Sucrose $1 \%$.

The cultures were incubated at $37{ }^{\circ} \mathrm{C}$ with an angle of 30 degrees. Biofilm formation was evaluated after 24-48 hours with 0 for no adhesion and 4 to strongly adhesive (Murchison et al., 1981). However this surface allows to quickly screen the biofilm formation ability of some strains only.

Dentin-Dentin discs both human and bovine origin have been used (Li et al., 2014)

Enamel- Enamel is mostly used substratum for cariogenic biofilm models. It may be of human or bovine origin (Van de Sande et al., 2011).

Polystyrene- Polystyrene microtiter plates provide a sterile abiotic surface for studying bacterial formation. The 96 well microtiter plate is used to study biofilms.

Hydroxyapatite- Saliva coated hydroxyapatite beads have been used in various studies. Dual biofilms of Streptococcus sobrinus, S.mutans and S.sanguinis, S.mutans and Veilonela parvula strains have been studied using this model (Kara et al., 2006; Rozen et al., 2001; Li et al., 2014).

Non cultivable methods to study oral Biofilms

In Situ Hybridization Fluoresence (FISH).
Multiple species of microbes can be detected in biofilm sample using FISH (Thurnheer et al., 2004). It is possible to perform several consecutive FISH procedures with multiple rRNA to identify simultaneously many members of biofilm (Almstrand et al., 2013).

\section{Confocal Laser Scanning Microscopy (CLSM)}

CLSM enables to observe the biofilms in 3 dimensions. High quality biofilm images are collected and visualised. Briefly a 15 minutes dark incubation period is given, an excitation wavelength of $488 \mathrm{~nm}$ to collect all light emitted between 500 and $550 \mathrm{~nm}$ and over $560 \mathrm{~nm}$ by various filters is used. The scan mode time series of time lapse scans at intervals of $10 \mathrm{~s}$ during $590 \mathrm{~s}$ in continuous scanning mode with $10 \mathrm{X}$ objective lens is then used (De et al., 2012). The distribution of live and dead cells at the different biofilm development times can also be investigated by this method.

\section{Scanning Electron Microscopy (SEM)}

In scanning electron microscopy biofilms are fixed for 24 hours in a $2.5 \%$ glutraldehyde solution and dehydrated with a graded ethanol series, subjected to a critical point drying with $\mathrm{CO}_{2}$, covered with gold $(10 \mathrm{~nm})$ and examined under SEM. SEM has been used to study the role of red complex bacteria in the colonization of gingival epithelia by sub gingival biofilms, multispecies biofilms have been studied by this method (Standar et al., 2010; Thumheer et al., 2014)

\section{Polymerase Chain Reaction (PCR)}

PCR is the most conventionally used molecular biology approach to identify and count bacterial species in oral biofilms in oral diseases like caries and periodontitis. PCR based methods have been used in single or 
multiplex approaches which involve amplification and sequencing of fragments of the 16S rRNA gene to detect bacterial species associated with periodontal disease or caries (Porphyromonas gingivalis, Tannerella forsythia and Treponema denticola (Elick and Pfister, 2002; Garcia et al., 1998; Rocas et al., 2001). Quantitative real-time PCR is used to observe microbial shifts in caries and periodontitis models (Zaura et al., 2011; Karched et al., 2015). However the limitation of the q PCR is its inability to discriminate between live and dead cells.

\section{Checkerboard DNA-DNA hybridization}

New molecular methods include application of DNA probes specific to oral bacterial species in checkerboard DNA- DNA hybridization, which initially allowed the detection and enumeration of bacteria species from around 43 biofilm samples simultaneously (Socransky et al., 1994). Using this technique around 40 taxa in supragingival and subgingival plaque samples it was found that Actinomyces species is the most established species at oral sites (Ximenz-Fyvie et al., 2000).

Table.1 The diversity of human oral microbiome (Human Oral Microbiome Database website, www.homd.org)

\begin{tabular}{|l|}
\multicolumn{1}{|c|}{ Phylum } \\
\hline Actinobacteria \\
\hline Bacteroidetes \\
\hline Chlamydiae \\
\hline Chlorobi \\
\hline Chloroflexi \\
\hline Cyanobacteria \\
\hline Euryarchaeota \\
\hline Firmicutes \\
\hline Fusobacteria \\
\hline Gracilibacteria (GN02) \\
\hline Proteobacteria \\
\hline SR1 \\
\hline Saccharibacteria (TM7) \\
\hline Spirochaetes \\
\hline Synergistetes \\
\hline WPS-2 \\
\hline
\end{tabular}

\section{S rRNA PCR cloning methodology}

Because not all microbes can grow in culture medium, a culture independent technique started with the implementation of $16 \mathrm{~S}$ rRNA PCR cloning sequencing methodology amplifying the $16 \mathrm{~S}$ rRNA genes directly from samples. Amplicons were then cloned and sequenced. Many studies have used this

\begin{tabular}{|c|c|c|}
\hline $\begin{array}{c}\text { Number of genomes in } \\
\text { the phylum }\end{array}$ & $\begin{array}{c}\text { Number of taxa in } \\
\text { the phylum }\end{array}$ & $\begin{array}{c}\text { Number of taxa } \\
\text { with genomes }\end{array}$ \\
\hline 222 & 118 & 94 \\
\hline 135 & 125 & 63 \\
\hline 5 & 1 & 1 \\
\hline 0 & 3 & 0 \\
\hline 4 & 3 & 1 \\
\hline 1 & 1 & 1 \\
\hline 1 & 1 & 1 \\
\hline 699 & 266 & 167 \\
\hline 54 & 37 & 22 \\
\hline 2 & 5 & 5 \\
\hline 434 & 141 & 89 \\
\hline 1 & 5 & 1 \\
\hline 7 & 19 & 5 \\
\hline 35 & 50 & 8 \\
\hline 8 & 8 & 3 \\
\hline 0 & 1 & 0 \\
\hline
\end{tabular}

technique to identify oral microbiota profiles in health and disease (Riggio et al., 2008; Tanner et al., 2011). This has led to the development of Human oral microbiome database (Human Oral Microbiome Database, 2012) which aims to identify and catalogue all the species found in oral cavity. More rapid identification of human oral bacterial $16 \mathrm{~S}$ rRNA gene sequences as compared to other 
methods has been used recently in CORE $16 \mathrm{~S}$ rRNA DNA database (Griffen et al., 2011).

\section{Next generation sequencing methods}

The next generation sequencing methods are based on the detection of pyrophosphate released during DNA synthesis. The DNA libraries are constructed from the sample and the DNA fragments are amplified within an emulsion and sequencing done. The nucleotide sequence data is then read (Ronaghi, 2001). The data is then processed through bioinformatics, compared with sequences from available databases like Human Oral Microbiome database or the ribosomal database project (Cole et al., 2009).

Many studies of oral biofilm have been done using this method. Saber et al., (2012) have seen that Streptococci, Actinomyces and unknown taxa are present in the microbiota of symptomatic periapical lesions. Another study by Ahn et al., (2011) determining the oral microbiome profile in oral cancer volunteers showed the presence of Firmicutes, Proteobacteria, Bacteriodetes, Acinobacteria and Fusobacteria. Pyrosequencing provided better sensitivity in identification of the bacteria.

The drawback of this technique is that the sequencing errors result in overestimation of microbial diversity and closely related species cannot be differentiated. The basic principles of biofilm formation are now known but the molecular understanding of the formation and structural organisation of various types of biofilms has just begun to emerge (Bjarnsholt et al., 2018).

\section{Limitations of molecular analysis of oral biofilms}

Molecular methods of analysis of oral biofilms are expensive, time consuming and require high level of expertise to process the data. The inability of this technique to use in clinical setting limits their use in diagnostics. The polymicrobial nature of oral diseases limits the use of oral biofilms to be used in chairside tests as diagnostic tools. However, they have a potential in, "minimally invasive dentistry".

\section{Control of pathogenic bacteria in the oral biofilm}

The pathogen associated biofilms can evade human host defences and are frequently associated with persistent infections, resistant to antibiotic therapy (Kostakioti et al., 2013). The concentrations of antibiotics needed to eradicate a biofilm are in the range of 1001000 times more than minimum inhibitory concentration (MIC) (Hoiby et al., 2010). A combination of antimicrobial peptides and essential oils, biofilm degrading enzymes, quorum sensing inhibitors and nanoparticles as potential biofilm agents have been used (Algburi et al., 2017). Recently bacteriocins which are antimicrobial peptides produced by bacteria typically $2-10 \mathrm{KDa}$ in size generally targeting closely related bacteria particularly the class I bacteriocin known as lantibiotics are being used (Mathur et al., 2018). S.mutans is an important organism causing dental caries. Several studies have been attempted to target S.mutans to see effect of various reagents on S.mutans biofilms (Clark et al., 2017). Antibiofilm activity against Enterococcus faecalis another biofilm forming organism in the oral cavity has also been attempted. E.faecalis biofilms have reduced levels of extracellular polysaccharides as compared to S.mutans biofilms. Actinomyces viscosus is frequently found as a biofilm former in the periodontal pockets (Balto et al., 2015). Like E.faecalis, it is also implicated in endocarditis. Studies have been reported to control the biofilm of this organism. Candida albicans biofilms 
associated with oral thrush have also been targeted for disruption of hyphal formation essential for $C$. albicans biofilm formation (Graham et al., 2017).

The oral microbiome is diverse with multispecies microbial communities in oral surfaces in structurally and functionally organised biofilms. Biofilm infections are challenging to treat and antibiotic therapy poses problems of resistance. Since biofilm infections contribute upto $80 \%$ of human microbial infections and the oral biofilms associated with caries, periodontitis and diseases like oral cancer. The functional and structural organisation of oral biofilms needs to be studied in much detail.

\section{References}

Ahn, J; Yang, L; Paster, B; et al., 2011. Oral microbiome profiles: 16SrRNA pyrosequencing and microarray comparison. PLoS One, 6(7); e22788.

Algburi, A; Comito, N; Kashtanov, D; Dicks, LM; Chikandas, ML; 2017. Control of biofilm formation, antibiotics and beyond. Appl Environ Microbiol, 83; e02508-e02516.

Almstrand, R; Daims, H; Persson, F; Sorrensson, F; Hermansson, M; 2013. New methods for analysis of spatial distribution and coaggregation of microbial populations in complex biofilms. Applied and Environmental Microbiology, 79 (19); 5978-5987.

Ammann, TW; Belibsakis, GN; Thurnheer, T; 2013.Impact of early colonizers on in vitro biofilm formation. PLoS ONE, 8(12); e0083090.

Badger, JH; Ng, PC; Venter, JC; 2011. The human genome, microbiomes and disease. In; Nelson KE, ed. Metagenomics of the human body. Springer Science: New York, 17; 1-14.
Balto, HA; Shakoor, ZA; Kanfar, MA; 2015. Combined effect of a mixture of tetracycline, acid and detergent and nisin against Enterococcus faecalis and Actinomyces viscosus biofilms. Saudi Med J, 36; 211-215.

Bik, EM; Long, CD; Armitage, GC; 2010 et al., Bacterial diversity in the oral cavity of ten healthy individuals. ISMEJ, 4; 962-974.

Bjarnsholt, T; Bahlin, K; Dufrene, YF; Gomelsky, M; Maroni, A; Ramstedt, M; Rumbaugh KP; Schulte, T; Sun, L; Akerlund, B; Romling, U; 2018. Biofilm formation - what we can learn from recent developments, $\mathbf{J}$ Intern Med, (6). doi:10.111/join.12782.

Clark, SA; Vinson, L; Eckert, G; Gregory, RL; 2017. Effect of commonly prescribed medications on S.mutans biofilm. An in vitro study. J.Clin.Pediatr.Dent, 41; 141-146.

Cole, J; Wang, Q; Cardenas, E et al., 2009. The Ribosomal Database Project: improved allignments and new tools for rRNA analysis. Nucleic Acid Res, 37; D141-D145.

De, F G, Carvalho; Puppin -Rontani, RM; SBP de Fucio; T de Cassia Negreni; Carlo, HL; Garcia-Godoy, F; 2012. Analysis by confocal laser scanning microscopy of the MDPB bactericidal effect on S.mutans biofilm CLSM analysis of MDPB bactericidal effect on biofilm. Journal of applied Oral Science, 20(5); 568-575.

Dewhirst, FE; Chen, T; Izard, J et al., 2010. The human oral microbiome. J Bacteriol. 192, 5002-5017.

Drescher, J; Schlafer, S; Schaudinn, C; 2010 et al., Molecular epidemiology and spatial distribution of Selemonas spp in subgingival biofilms. Eur J oral Sci, 118; 466-474.

Elick, S; and Pfister, W; 2002. Comparison of microbial cultivation and a commercial 
PCR based method for detection of Periodontopathogenic species in subgingival plaque samples. J Clin Periodontol, 29(7); 638-644.

Em, B R, Long CD; Armitage, GC et al., 2010.Bacterial diversity in the oral cavity of ten healthy individuals. ISMEJ, 4:962-974.110.1038/ismej, 30.

Gaffen, SL; Hersberg, MC; Taubman, MA; Van Dyke, TE; 2014. Recent advances in host defense mechanisms/therapies against oral infectious diseases and consequences for systemic diseases. Adv Rent Res, 26:3037.doi.10.1177/0022034514525778.

Garcia, L; Tercero, J; Legido, B; Ramos, J; Alemany, J; Sanz, M; 1998. Rapid detection of Actinobacillus actinomycetemcomitans, Prevotella intermedia and Poryphyromonas gingivalis by multiplex PCR. J Periodontol Res, 33(1); 59-64.

Graham, CE; Cruz, MR; Garsin, DA; Lorenz, MC; 2017. Enterococcus faecalis bacteriocin Ent V inhibits hyphal morphogenesis, biofilm formation and virulence of Candida albicans. Proc Natl Acad Sci USA, 114; 4507-4512.

Griffen, A; Beace, C; Firestone, N; et al., 2011. CORE: a phylogenetically curated $16 \mathrm{~s}$ rDNA database of the core oral microbiome. PLoS One, 6(4); e19051.

Guggeheim, B; Egiertsen, P; Schupbach, S; Shapiro; 2001. Validation of an in vitro biofilm model of supragingival plaque. J of dental Res, 80(1); 363-370.

Hajishengallis, G; 2015. Periodontitis: from microbial immune subversion to systemic inflammation. Nat Rev Immunol, 15; 30-44. doi:10.1038/ nri3785.

Hannig, M; 1999. Ultrastructural investigation of pellicle morphogenesis at two different oral sites during a $24 \mathrm{hr}$ period. Clin Oral Investig.3, 88-95.
Hoiby, N; Ciofu, O; Bjarnsholt, T; 2010. Pseudomonas aeruginosa biofilms in cystic fibrosis. Future Microbiol, 5; 1663-1674.

Human Oral Microbiome Database; 2012. Human oral microbiome database at the Forsyth Institute (USA). Available from http://www.homd.org.acessed January, 2013.

Jiao, Y; Hasegava, M; Inohara, N; 2014. The role of oral pathbionts in dysbiosis during periodontitis development. J Dent Res, 93; 539-546.

Kara, D; Luppens, SBI; Cate, JMT; 2006. Difference between single and dualspecies biofilms of Streptococcus mutans and Veillonella parvula in growth, acidogenicity and susceptibility to chlorhexidine. Euro $\mathbf{J}$ of oral sciences, 114(1); 58-63.

Karched, M; Bhardwaj, RG; Inbamani, A; Arikainen, S; 2015. Quantification of biofilm and planktonic lifeforms of coexisting periodontal species. Anaerobe, 35; 13-20.

Kim, J; and Amar, S; 2006. Periodontal disease and systemic conditions; a bi directional relationship. Odontology; 94, 10-21.doi:10.1007/S10266-0060060-6.

Klinke, T; Guggenheim, B; Klimm, W; Thurnheer, T; 2011. Dental caries in rats associated with Candida albicans. Caries Res, 45; 100-106.

Kolenbrander, PE; Anderson, RN; Blehert, DS; Egland, PG; Foster, JS; Palmer, RJ; 2002. Communication among oral bacteria.Microbiol Mol Biol Rev, 66; 486-505.

Kolenbrander, PE; Palmer, RJ; Periamy, S; Jakubovics, NS; 2010. Oral multispecies biofilm development and the key role of cell-cell distance. Nature Rev Microbiol, 8; 471480.doi.1038/nrmicro 2381. 
Kostakioti, M; Hadjifrangiskou, M; Hultgren, SJ; 2013. Bacterial biofilms development, dispersal and therapeutic strategies in the dawn of post antibiotic era. Cold Spring Harb Perspect Med, 3; a010306.

Lamont, RJ; and Hajishengallis, G; 2014. Polymicrobial synergy and dysbiosis in inflammatory disease. Trends Mol Med, 21 ;

$172-$

183.doi:10.1016/j.molmed.2014.11.004.

Li M, Huang; R, Zhou X; Zhang, K; Zheng, $\mathrm{X}$; Gregory, RL; 2014. Effect of nicotine on dual species biofilms of Streptococcus mutans and Streptococcus Sanguinis. FEMS Microbiology Letters, 350(2); 125-132.

Li, Y; Carrera, C; Chen, R; 2014 et al., Degradation in the dentin-composite interface subjected to multispecies biofilm challenges. Acta Biomaterialia, 10(1); 375-383.

Mark Welch, JL; Rossetti, BJ; Rieken, CW; Dewhirst, FE; Borisy, GG; 2016. Biogeography of a human oral microbiome at the micron scale. Proc. Natl Acad Sci. U.S.A., 113; E791E800.

Marloes, Lof; Marleen, MJ; Krom, BP; 2017. Metabolic interactions between bacteria and funnngi in commensal oral biofilms. J. Fungi, 3; 40.

Marsh, PD; 1994. Microbial ecology of dental plaque and its significance in health and disease. Adv Dent Res, 8; 263-271.

Marsh, PD; 2010. Microbiology of dental plaque biofilms and their role in oral health and caries. Dent Clin North Am, 54; 441-454.

Marsh, PD; and Bradshaw, DJ; 1995. Dental Plaque as a biofilm. $\mathbf{J}$ of Indust Microbio, 15(3); 169-175.

Marsh, PD; and Zaura, E; 2017. Dental biofilm: ecological interactions in health and disease. J Clin Periodontol; 18 S12S22.
Mathur, H; Field, D; Rea, MC; Cotter, PD; Hill, C; Ross, RP; 2018. Fighting biofilms with lantibiotics and other groups of bacteriocins. Biofilms and Microbiomes, 4; 9.

Murchison, H; Larrimore, S; Curtiss, R; 1981. Isolation and characterization of Streptococcus mutans mutants defective in adherence and aggregation. Infect and Immun, 34(3); 1044-1055.

Nelsen-Filho, P; Borba, I G; Mesquita, KS; Silva, RA; Quiroz, AM; Silva, IA; 2013. Dynamics of the microbial colonization of the oral cavity in new borns. Braz Dent J, 24; 415-419.

Pihlshrom, BL; Michalowicz, BS; Johnson, NW; 2005. Periodontal diseases. Lancet, 366; 1809-1820.

Riggio, M; Lennon, A; Rolph, H et al., 2008. Molecular identification of bacteria on the tongue dorsum of subjects with and without halitosis. Oral Dis, 14(3); 251258.

Rocas, I; Siqueira, JJ; Santos, K; Coelho, A; 2001. Red Complex (Bacteroides forsythus, Poryphyromonas gingivalis and Treponema denticola) in endodontic infections. A molecular approach. Oral Surg oral Med oral pathol oral Radiol Endod, 91(4); 468471.

Ronaghi, M; 2001. Pyrosequencing sheds light on DNA sequencing. Genome Res, 11(1); 3-11.

Rozen, R; Bachrach, G; Zachs, B; Steinberg, D; 2001. Growth rate and biofilm thickness of Streptococcus sobrinus and Streptococcus mutans on hydroxapatite. Acta pathologica Microbiologica et Immunologica Scandinavica, 109(2); 155-160.

Saber, M; Schwarzberg, K; Alonaizen, F; et al., 2012. Bacterial flora of dental periadicular lesions analysed by the 454-pyrosequencingtechnology.J Endod, 38(11); 1484-1488. 
Shchipkova, AY; Nagaraja, HN; Kumar, PS; 2010. Subgingival microbial profiles of smokers with periodontitis. J Dent Res, 89; 1247-1253.

Socransky, S; Smith, C; Martin, L; Paster, B; Dewhirst, F; Levin, A; 1994. "Chekerboard" DNA -DNA hybridization. Biotechniques, 17(4; 788-792.

Standar, K; Kreikemeyer, B; Redauz, S; Munter, WL; Laue, M; Podbielski, A; 2010. Setup of an in vitro test system for basic studies on biofilm behaviour of mixed species cultures with dental and periodontal pathogens. PLOS One, 5(10); e13135.

Stephens, C; 2002. Microbiology: Breaking down Biofilms. Curr Biol, 12; R132134.

Tanner, A; Mathney, J; Kent, R et al., 2011. Cultivable anaerobic microbiota of severe early childhood caries. J Clin Microbiol, 49(4); 1464-1474.

Tanner, AC; Mathney, JM; Kent, RL et al., 2011. Cultivable anaerobic microbiota of severe early childhood caries. J Clin Micro Biol, 49; 1464-1474.

Thumheer, T; Belibasakis, GN; Bostanci, N; 2014. Colonisation of gingival epithelia by subgingival biofilms in vitro: role of red complex bacteria. Archives of Oral Biology, 59(9); 977-986.

Thurnheer, T; Gmur, R; Guggenheim, B; 2004. Multiplex FISH analysis of a six species bacterial biofilm. Journal of Microbiological Methods, 56(1); 37-47.

Van de Sande, FH; Azevedo, MS; Lund, RG; Heysmans, M.C. D; Cenci, MS; 2011. An in vitro biofilm model for enamel demineralization and antimicrobial dose-response studies. Biofouling, 27(9); 1057-1063.

Wade, WG; 2013. The oral microbiome in health and disease. Pharmacol Res, 69; $137-$ 143.doi.10.1016/j.phrs.2012.11.006.

Wilson, M; 2005. Microbial inhabitants of humans: their ecology and role in health and disease. Cambridge University Press: Cambridge.

Ximenz-Fyvie, C; Haffajee, A; Socransky, S; 2000. Microbial Composition of supra and subgingival plaque in subjects with adult periodontitis. J Clin Periodontol, 27(10); 722-732.

Zaura, E; Buijs, MJ; Hoogenkamp, MA et al., 2011. The effects of fractions from shiitake mushroom on composition and cariogenicity of dental microcosms in an in vitro caries model. Journal of Biomedicine and Biotechnology, 10; e 135034.

Zaura, E; Keijser, BJ; Huse, SM; Crielard, W; 2009. Defining the healthy, "Core Microbiome" of oral microbial communities, BMC Microbial. 259, 12.

\section{How to cite this article:}

Sonia B. Bhardwaj. 2018. Oral Biofilms. Int.J.Curr.Microbiol.App.Sci. 7(11): 643-652. doi: https://doi.org/10.20546/ijcmas.2018.711.078 